International Journal of Mechanical Engineering and Technology (IJMET)

Volume 11, Issue 12, December 2020, pp. 19-32, Article ID: IJMET_11_12_003

Available online at https://iaeme.com/Home/issue/IJMET?Volume $=11 \&$ Issue $=12$

ISSN Print: 0976-6340 and ISSN Online: 0976-6359

DOI: https://doi.org/10.34218/IJMET.11.12.2020.003

(C) IAEME Publication

Scope Database Indexed

\title{
LIQUID COOLING FOR 3D MICROPROCESSORS PROCESSORS
}

\author{
Pinaknath Dewanji \\ Engineering, The University of Texas at Arlington, Arlington, Texas, USA.
}

\begin{abstract}
In today's world 3D ICs and microprocessors are the latest technique and most promising way that the total wire length and interconnect lengths can be reduced dramatically. But without a way to efficiently cool these 3D IC they suffer significantly due to major heat accumulation in between the layers which effects its reliability and performance. This can cause the Chip to fail. In this project we take a look at the advantages of liquid cooling for 3D microprocessors. We also evaluate the leakage power consumption and the lifetime reliability depending on the temperature of each functional unit in the $3 D$ multi-core processor along with modelling and dynamic management of multicore systems and look at the liquid cooling setups for CPU, along with a quick glance at the future of liquid cooling which involves a complex interconnected layout of both Fluidic and electrical TSVs to design these modern $3 D$ ICs and microprocessors.
\end{abstract}

Key words: Three dimensional Processors, Liquid cooling, Thermal management, Microchannel, Throughsilicon vias.

Cite this Article: Pinaknath Dewanji, Liquid Cooling for 3D Microprocessors Processors, International Journal of Mechanical Engineering and Technology. 11(12), 2020, pp. 19-32.

https://iaeme.com/Home/issue/IJMET?Volume=11\&Issue=12

\section{INTRODUCTION}

Conventionally in 2D Microprocessors, cooling was performed by attaching a heat sink on the package and removing heat via fan or fins, even by reducing the interconnect junction resistance by using thermal compound has aided in this pursuit. As technology scales down and the densities of integration increases, interconnect delay becomes a great concern and the main performance factor. To reduce these delays $3 \mathrm{D}$ integration has been a major focus since vertically stacked processors enable faster on chip communication and enable reduces power draw by the chip. But this 3D stacking causes significant increases in heat density on the chip due to the close stacking of the power dissipating components, which delays the heat flow from the die to the heat sink in conventional air cooling, so without efficient cooling method inside the package, 3D chips will suffer substantial performance degradation by DTM (Dynamic 
Thermal Management). To accomplish this task, thermally driven floor planning, Dynamic voltage frequency (DVFS), temperature aware allocation and thread mitigation are some techniques used. Considering the high-power densities in 3D systems a shift from air to liquid cooling provides huge benefits, it is done by fabricating micro channels in the substrate, also by fabricating microchannels within the interface materials between the layers of the 3D architecture. Then a coolant is pumped through the microchannel to remove heat. It has been demonstrated that interlayer reduced heat densities of $25 \mathrm{~W} / \mathrm{cm}^{2} \sim 50 \mathrm{~W} / \mathrm{cm}^{2}$ and that a layer of integrated microchannel cooling with water reduced heat density up to $135 \mathrm{~W} / \mathrm{cm}^{2}$ within the 3D system [1].

\section{RELATED WORKS}

\subsection{D Integration Techniques}

3D integration consists of two different ways, die bonding techniques and MLBS (Multi Layered Buried System). Die binding is used for 2D system with TSV bonding. Whereas MLBS can be used to bond multiple multi planar dies, it is also possible to bond Many Heterogenous dies, thus bonding dissimilar technologies like DRAM \& CMOS Chips [1]. TSV integration techniques are used to connect multiple chip layers either mostly for electrical connections by passing vertically through the silicon die. This reduces latency and increases chip performance efficiency, but this can also be used for Cooling since the height for these TSV are very small and can integrate interlayer cooling techniques [1]. Also, smart scheduling choices are also a popular way to manage the heat from 3D stacks, but a ceiling has occurred where it can no longer provide enough cooling to help increase its complexity or performance by solely using this method, so liquid cooling is the future approach that is being widely considered in the research and consumer industry today.

\subsection{Liquid Cooling Techniques}

Using water as coolant is the most efficient way of cooling due to its large capacity to carry heat. Two most common methods are Direct cooling and Indirect cooling. In indirect cooling the coolant doesn't contact the electronic components, but micro channels are used instead. Whereas as in direct cooling can included methods like full immersion of the system or the chip itself. Compared to contemporary air cooling techniques using heat spreaders, this method is a far better technique to manage heat from 3D chips.

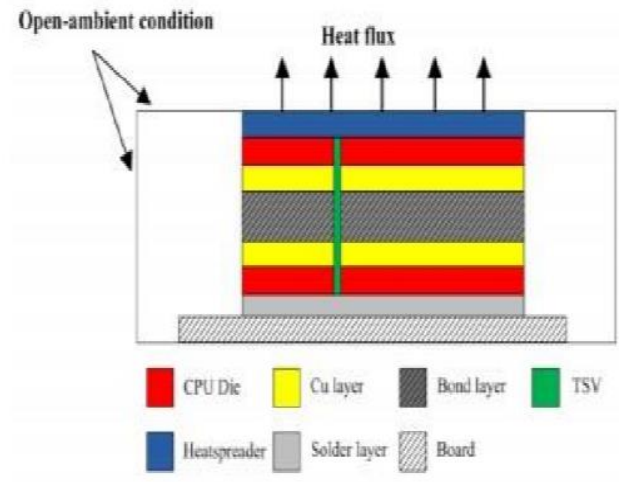

(a) Air cooling

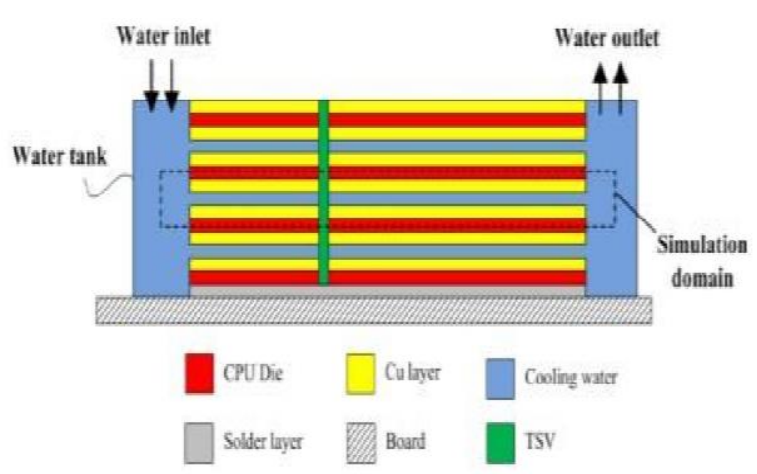

(b) Direct liquid cooling using water as a coolant

Figure .Liquid Cooling Techniques (a) and (b) 


\section{3D PROCESSORS WITH LIQUID COOLING MODELS}

\subsection{Radial Hierarchical Fluid Network}
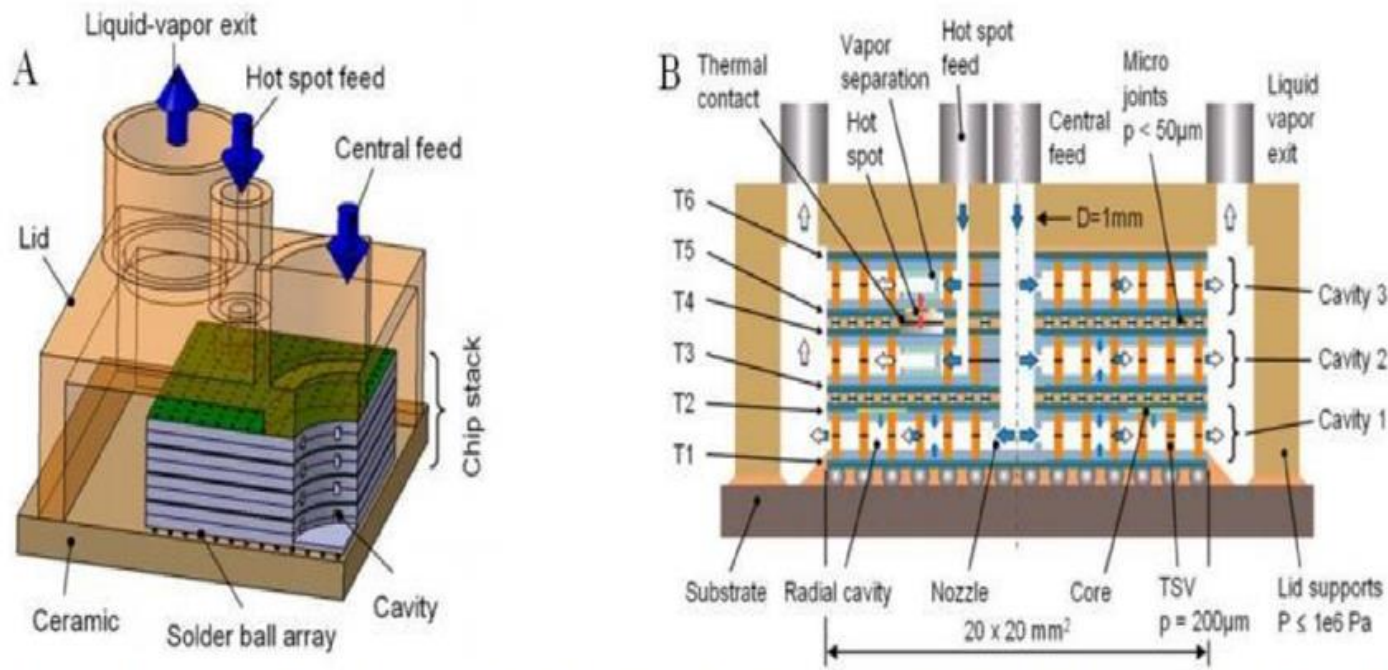

Figure 1: (A) Quadrant view of the chip stack module with refrigerant feed and peripheral vapor exit, (B) Module cross-section with six thermally active dies and three heat removal cavities.

A fluid port is introduced in the centre of the chip stack allowing the injected flow to radially expand and absorb heat along its path towards the peripheral outlet. Second, the bifurcation concept was implemented by incorporating an advanced branching network design including guiding walls to systematically split the flow, to mitigate flow by-passing. A comprehensive set of single cavity test chips were developed to systematically explore the building block elements presented earlier [2]. The first tranche of experiments involved the characterization of pin fin arrays with three different angles-of-attack (inline - PF0, staggered - PF45 and 27o PF27) followed by a multi-micro channel (PC) and a radial quadrant (RQ) test chip. Figure 4 depicts stereo microscopic images of the two-port PF27 and PF45 inlet-outlet plenum test chips [2]. The inlet plenum is $10 \mathrm{~mm}$ in width and $120 \mu \mathrm{m}$ in depth. Shown here are orifices with widths of $\mathrm{W} \sim 75 \mu \mathrm{m}$ (PF27) and $125 \mu \mathrm{m}$ (PF45) located upstream of the pin fin arrays which were etched into the cavity to suppress backflow into the inlet plenum [2]. The benchmarking of 13 single component refrigerants, namely R717, R600a, R290, R22, R32, R123, R125, R134a, R143a, R152a, R227ea, R245fa, R1234yf and R1234ze was performed by comparing the thermodynamic and transport properties of these fluids to select the base fluid for our application [2]. The benchmarking process considers the followings, i) moderate saturation pressure for acceptable mechanical load on the chip package, ii) low liquid-vapor density ratio for lower two-phase pressure drop, iii) high latent heat, iv) low fluid viscosity, v) lower surface tension for low nucleation onset and superheat, vi) relatively high liquid and vapor phase thermal conductivity, vii) low flammability level and viii) low ozone depletion (ODP) and global warming potential (GWP). After careful evaluation, R1234ze (trans1, 3, 3, 3tetrafluoroprop-1-ene) was selected as the primary cooling fluid [2]. 

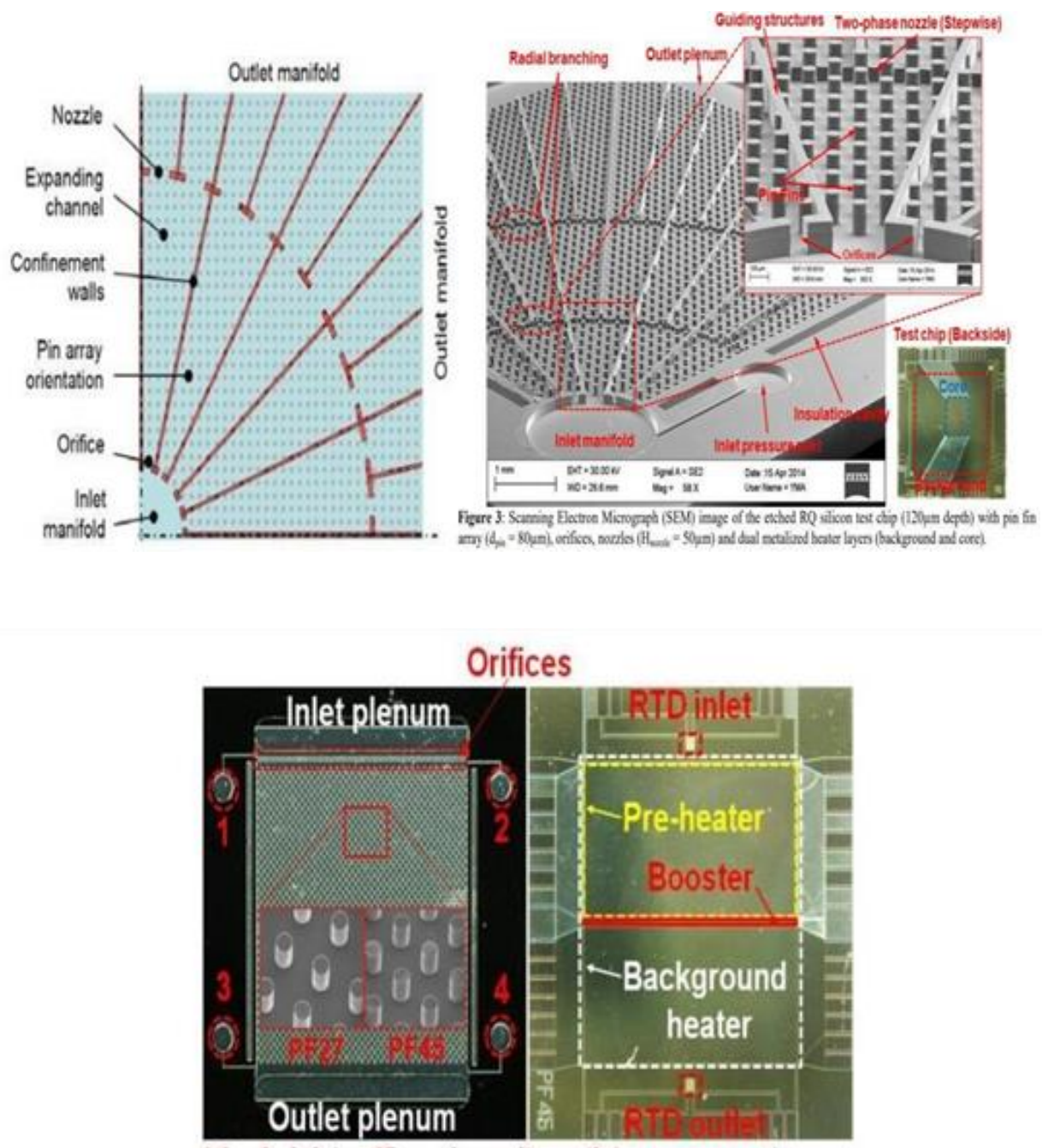

"1-4: Inlet, orifice, pin cavity, outlet pressure ports.

Figure 4: SEM images of the pin fin array orientations, (left) inline - PF0, (center) $27^{\circ}$ - PF27 and (right) staggered PF45.

Figure 13 presents the flow boiling results for the RQ test chip. The results presented here correspond to uniform heat flux conditions whereby the background heater was activated [2].

The manifold is equipped with 30(S1), 50.1(S2), 264(S3), 88(S4) and 30 $\mu \mathrm{m}$ (S5) wide orifices respectively as passive flow regulation devices.

Under low heat flux conditions, it was observed that two phase flow was triggered in subquadrants S1, S2, S4 \& S5 but not sub-quadrant S3 [2]. Dryout was encountered in sub-quadrant S5 at heat flux, $\mathrm{q}=32.8 \mathrm{~W} / \mathrm{cm} 2$ and a hot spot was observed (bottom right corner towards the outlet) [2]. A comparison of junction temperatures for various mass flow rates is shown in Figure 14. As expected, dryout occurred for the lowest mass flow rate of $\sim 0.52 \mathrm{~kg} / \mathrm{h}$ while a more uniform junction temperature profile was observed for the $0.8 \mathrm{~kg} / \mathrm{h}$ case (Figure 14(b) when two-phase flow was triggered in all subcavities [2]. In Figure 14(C), flow boiling was not triggered in S3 and a hot spot was systematically observed neat the outlet. So to conclude from this paper of radial quadrant test chip, dryout was always encountered in sub-quadrants with the smallest orifice gap (S5) under uniform heat flux conditions. Flow boiling was not triggered in sub-quadrant (S3) for some instances due to high flow rates along the cavity, resulting in hot spots due to lower single-phase heat transfer. An opposite trend was observed when flow boiling was triggered in this sub-cavity, resulting in a more uniform temperature profile across the entire chip under no-dryout conditions [2]. 

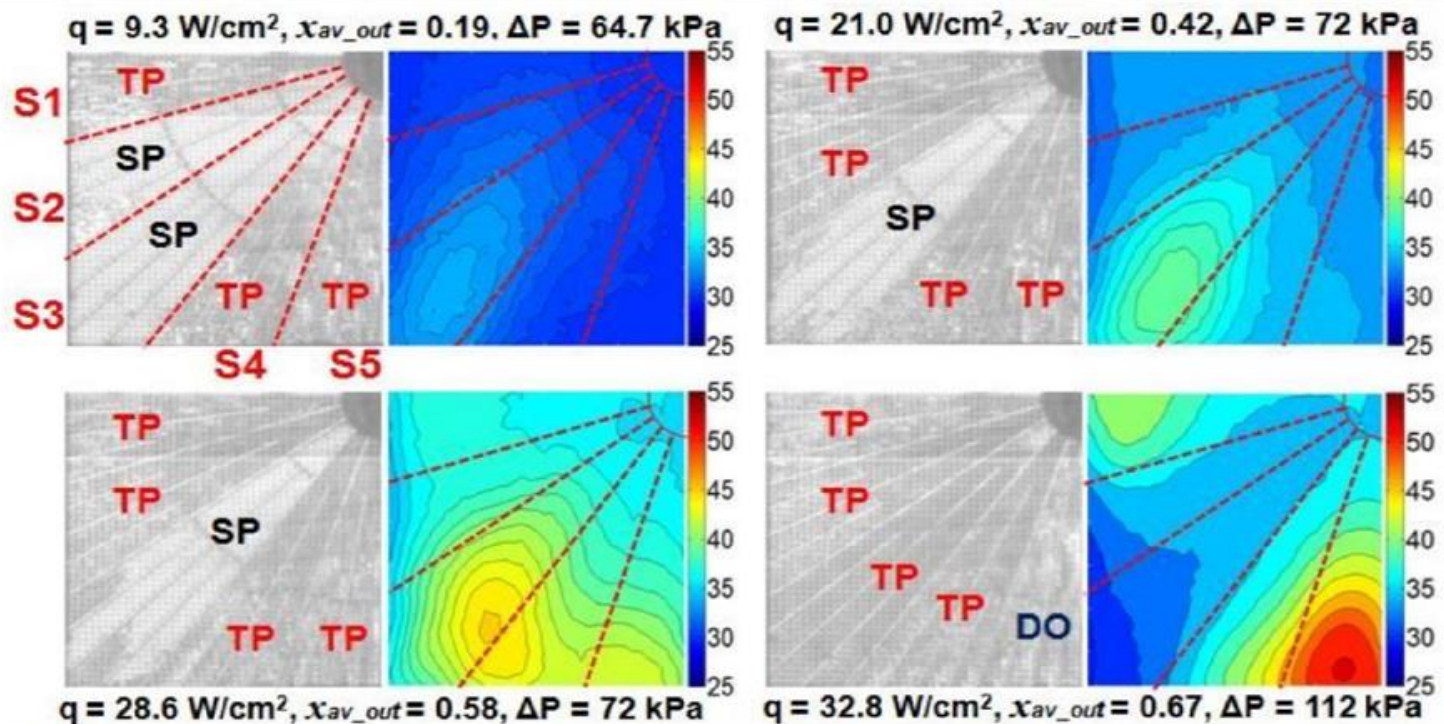

Figure 13: IR thermography of the RQ test chip for $\mathrm{T}_{\text {sat }}=30.4^{\circ} \mathrm{C}, \Delta \mathrm{T}_{\text {sub }} \sim 6.6 \mathrm{~K}, \mathrm{~m}=1.03 \mathrm{~kg} / \mathrm{h}$. $(\mathrm{SP}-$ single-phase, TP - two-phase, DO - dryout)

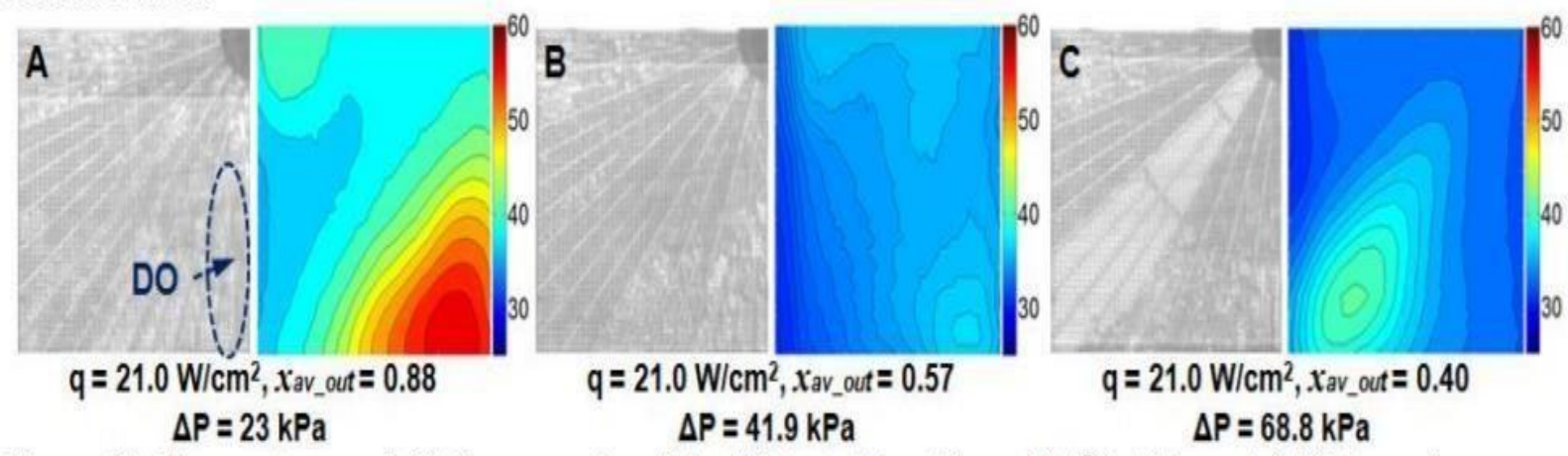

Figure 14: Flow patterns and IR thermography of the RQ test chip at $\mathrm{T}_{\text {sat }}=30.4^{\circ} \mathrm{C}, \Delta \mathrm{T}_{\text {sub }} \sim 4-6.5 \mathrm{~K}$ for various mass flow rates, (A) $0.52 \mathrm{~kg} / \mathrm{h}$, (B) $0.79 \mathrm{~kg} / \mathrm{h}$ and (C) $1.11 \mathrm{~kg} / \mathrm{h}$.

\subsection{Integrated Liquid Cooling Systems for 3-D Stacked TSV Modules}

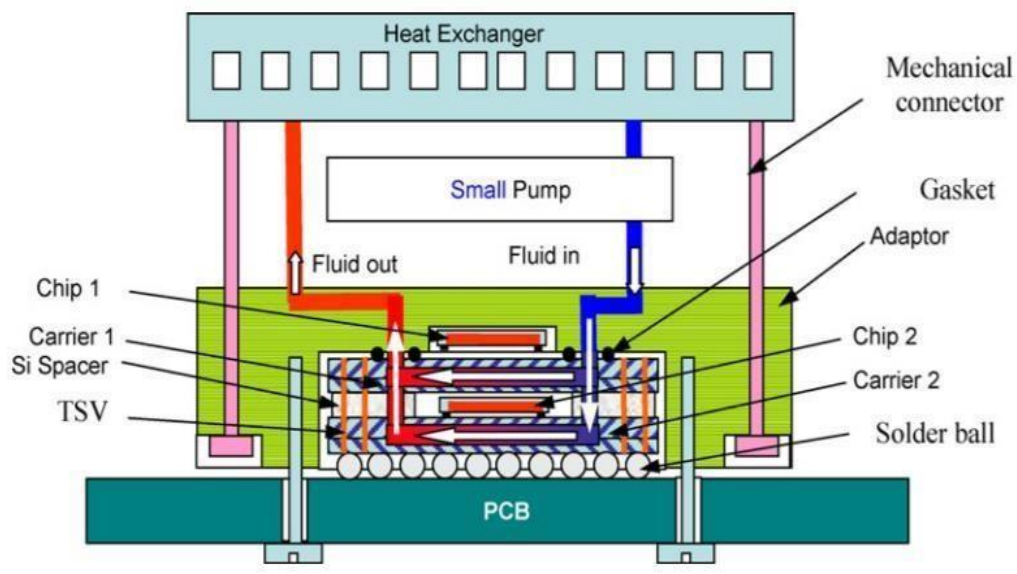

Fig. 1. Scheme of the integrated liquid cooling system for 3-D stacked module (nonscaled).

This proposed integrated cooling solution extracts the heat from a compact 3-D silicon module and dissipates it over a larger heat exchanger where conventional air cooling heat sinks suffice to extract the heat. The system is designed such that no external fluidic interconnects are required to assemble the package on the mother board [3]. The system is schematically shown 
in Fig. 1 which includes: 1) two silicon carriers $(15 \mathrm{~mm} \times 15 \mathrm{~mm})$ with fluidic microchannels embedded stacked vertically, and each carrier is mounted with a $10 \mathrm{~mm} \times 10 \mathrm{~mm}$ chip dissipating $100 \mathrm{~W}$ of heat [3]. A silicon spacer is assembled between these two carriers to house the chip and support the carriers; 2) an adaptor $(50 \mathrm{~mm} \times 50 \mathrm{~mm})$ for connecting the microchannels in the carriers and mini sized ports of the pump, which splits the flow from the outlets of the pump to the inlets of the microchannel carriers; 3) an external heat exchanger (50 $\mathrm{mm} \times 50 \mathrm{~mm}$ ) for heat removal to ambient/cold plate; and 4) a commercial small pump (50 mm $\times 55 \mathrm{~mm}$ ) with a maximum operation pressure of 2 bar and a maximum flow rate of $500 \mathrm{ml} / \mathrm{min}$ for driving the coolant [3]. The heated fluid is driven out from the stacked chip module to the heat exchanger by the pump, and the hot fluid is cooled down in the heat exchanger then the cooled fluid is driven back to the mo2) related works .
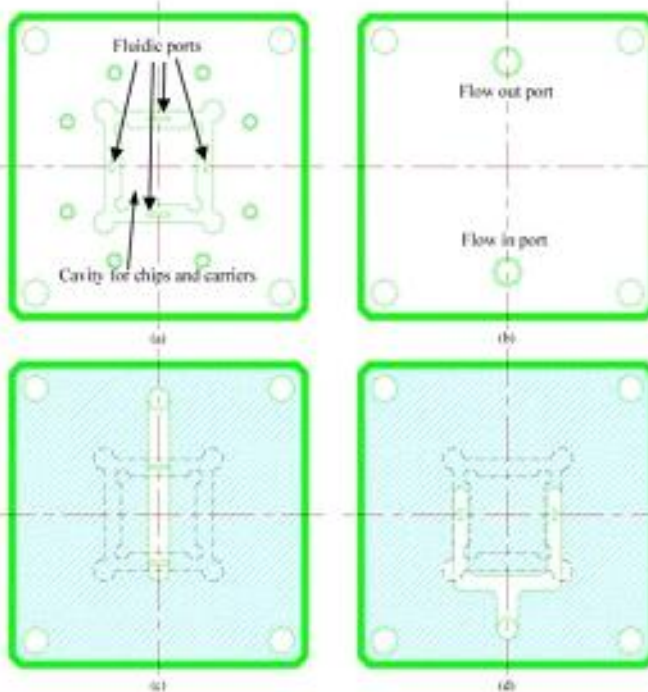
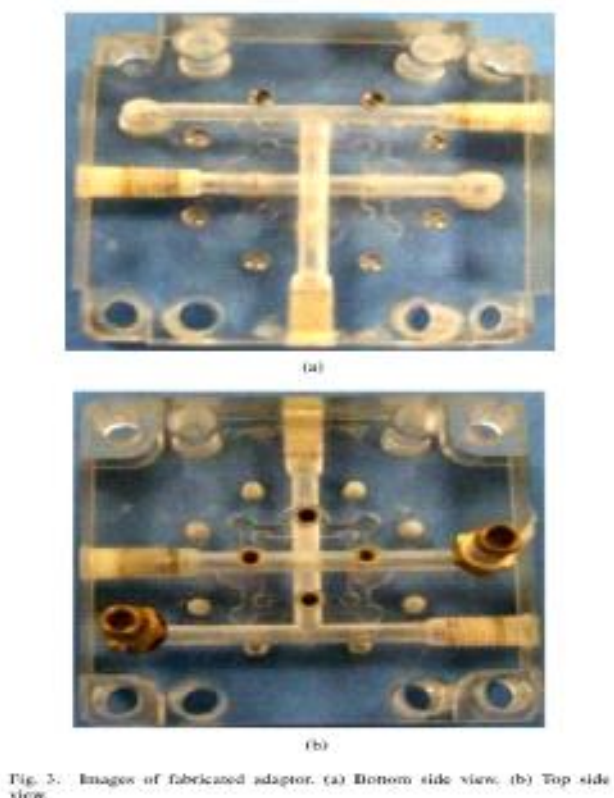

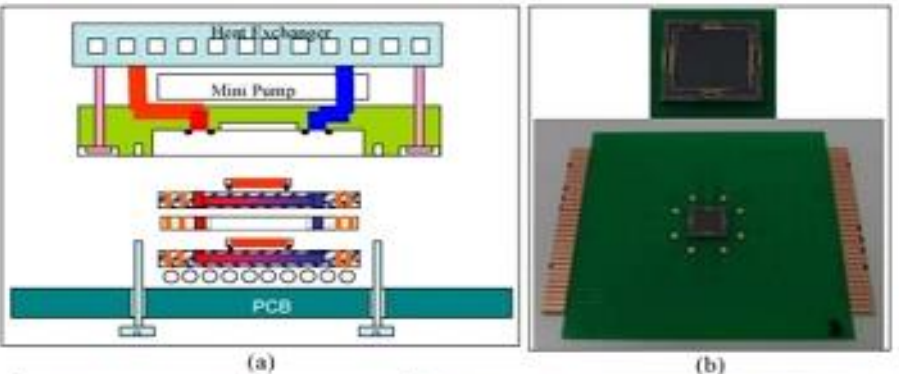

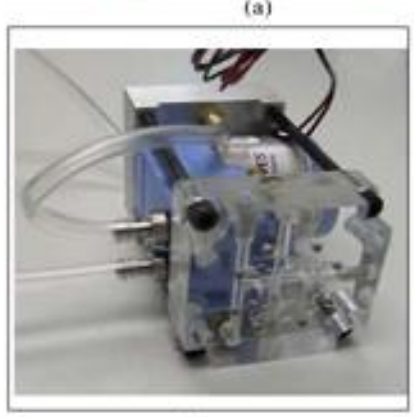

(c)

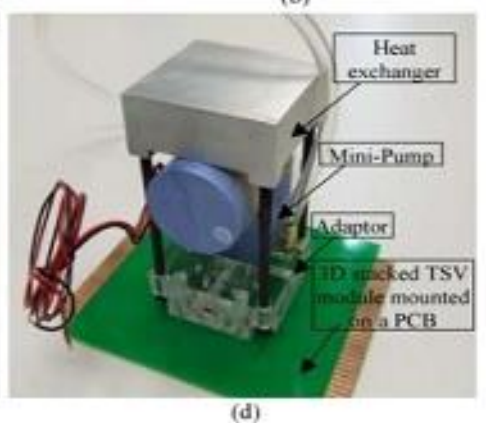

Fig. 8. Process of the integration for the liquid cooling system (a) schematic diagram, (b) image of assembled module, (c) image of assembled adaptor, mini-pump and heat exchanger, and (d) image of integrated enoting system.

When the flow rate in each carrier is $230 \mathrm{ml} / \mathrm{min}$ and the power of each chip is $100 \mathrm{~W}$, the thermal resistances for both carriers are about $0.18^{\circ} \mathrm{C} / \mathrm{W}$ [3]. However, the variation of the chip 
temperature for the optimized carrier is $8.1{ }^{\circ} \mathrm{C}$, which is much lower than that for the conventional carrier $\left(14.1^{\circ} \mathrm{C}\right)$ [3].

In Summary an integrated cooling solution for a 3-D silicon module developed has the following key features:

a. eliminating the bottlenecks of thermal interface material and spreading resistance;

b. forming fluidic interconnects connecting silicon micro-channel;

c. eliminating external fluidic circuits;

d. scale able to multiple number of 3-D stacks.

The Carrier Pressure drop accounts for around $87 \%$ of the system pressure drop. While the adaptor and heat exchanger only account for around $12.5 \%$ and $0.5 \%$ of the system pressure drop, respectively [3]. Such a distribution of the system pressure drop indicates that the hydraulic design of this system is efficient [3].

c) Integrated Microfluidic Cooling and Interconnects

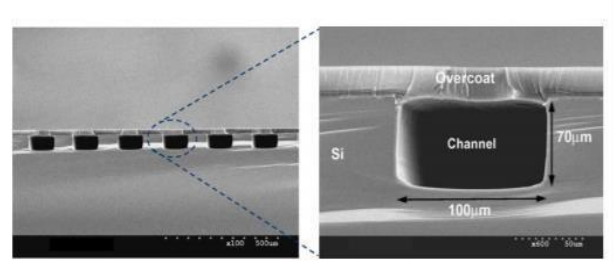

Fig. 5. Cross-sectional SEM micrographs of microchannels enclosed by monolithic sacrificial polymer method.

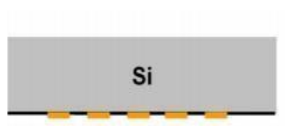

(a)

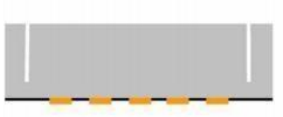

(b)

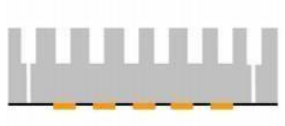

(c)

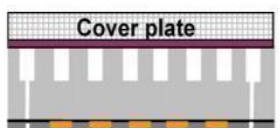

(d)

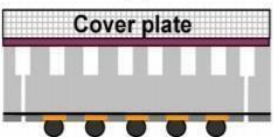

(e)

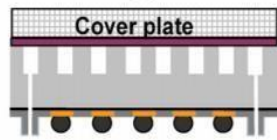

(f)
Fig. 6. Schematic of the fabrication process used to integrate the microchannel heat sink, the thermofluidic chip $\mathrm{I} / \mathrm{O}$ interconnections, and $\mathrm{C} 4$ solder bumps on a silicon die using wafer-scale batch processing: (a) the process begins after the completion of semiconductor BEOL processing, (b) through-wafer vias are patterned and partially etched, (c) trenches are patterned and simultaneously etched with the partially etched through-wafer vias, (d) microchannels are covered with glass or Si plate and a polymer passivation is deposited and pattern at Fig. 10. Schematic of the assembly process of a "microfluidic flip-chip:" the front-side, (e) solder bumps are deposited on the front-side using electrode- (a) alignment, (b) bonding using standard flip-chip bonders, (c) fluidic VO position, (f) polymer pipes are fabricated by a thick photodefinable polymer.

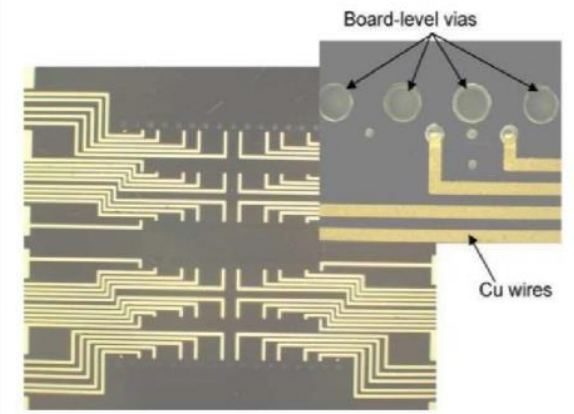

Fig. 9. Optical micrographs of test board substrates containing copper interconnects on the front-side and through-vias for the thermofluidic VO configuration in Fig. 8(b).
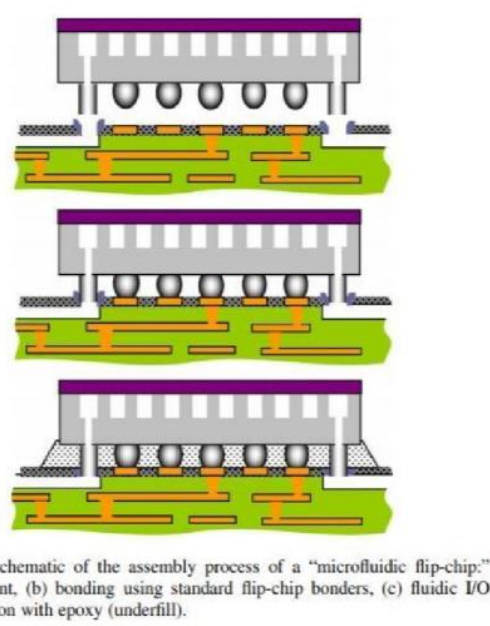

In addition to the microchannels, microfluidic interconnections to and from the microchannel heat sink are needed to enable on-chip microfluidic cooling. In this section, the concept of chip thermofluidic I/Os is introduced to enable chip-to-board fluidic interconnection. Fig. 6 illustrates a process used to fabricate and integrate the microchannel heat sink, the thermofluidic chip I/O interconnections, and $\mathrm{C} 4$ solder bumps on a silicon die using wafer-scale batch processing [4]. After fabrication, the polymer micropipes are cured at 160 for $1 \mathrm{~h}$. The passivation layer inside the pipes is next etched using wet etching to allow fluidic circulation. Following this process step, the wafer is diced. Based on this approach, wafer-level thermofluidic I/Os are formed and can be used to deliver the working fluid directly to the monolithically fabricated microchannel heat sink from board-level fluidic interconnects [4]. 
Two distinct substrate-level coolant delivery schemes are proposed and are illustrated in Fig. 8. In Fig. 8(a), the microfluidic channels are embedded on the front-side of the board substrate and orifices are formed through the overcoat. One challenge with the implementation of this scheme is that the available area on the chip-mounting side of the substrate is usually limited and occupied by signal routing [4]. To address this potential incompatibility, a second configuration was developed and is illustrated in Fig. 8(b). In this configuration, the manifold channels are embedded inside the multilayer board or fabricated at the opposite side of the board, and through-holes are utilized for fluidic interconnection. Fig. 9 illustrates the micrographs of the front-side of a silicon test substrate (silicon carrier) where a peripheral distribution of vias is fabricated and integrated with the electrical traces [4].

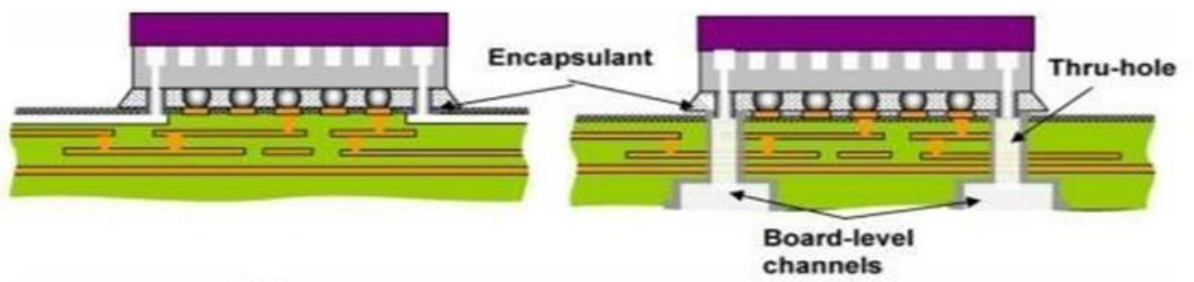

(a)

(b)

Fig. 8. Schematic of two distinct chip coolant delivery schemes to enable the on-chip microchannel heat sinks: (a) manifold channels are located at the frontside of the board, (b) manifold channels are located at the back-side of the board and large through-holes are used to interconnect with the micropipes, and thus, the on-chip microchannel heat sink.
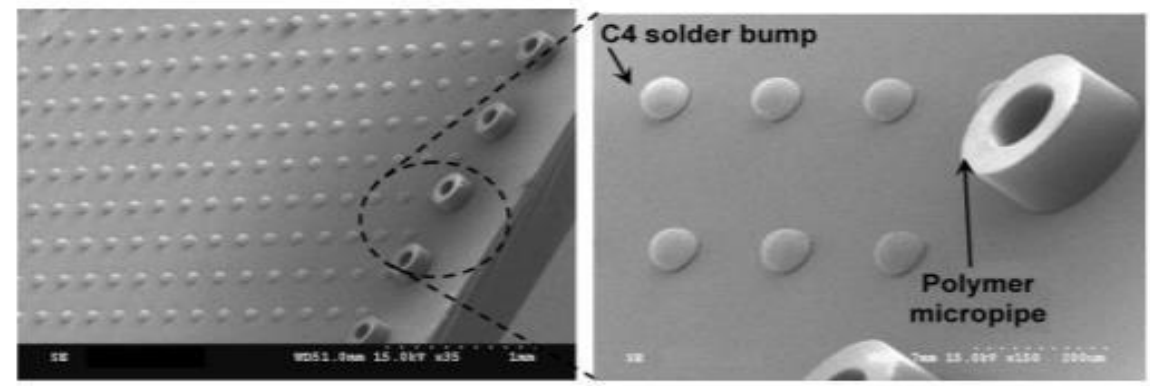

Fig. 7. SEM micrographs of a row of polymeric micropipes fabricated adjacent to an area-array distribution of solder bumps.

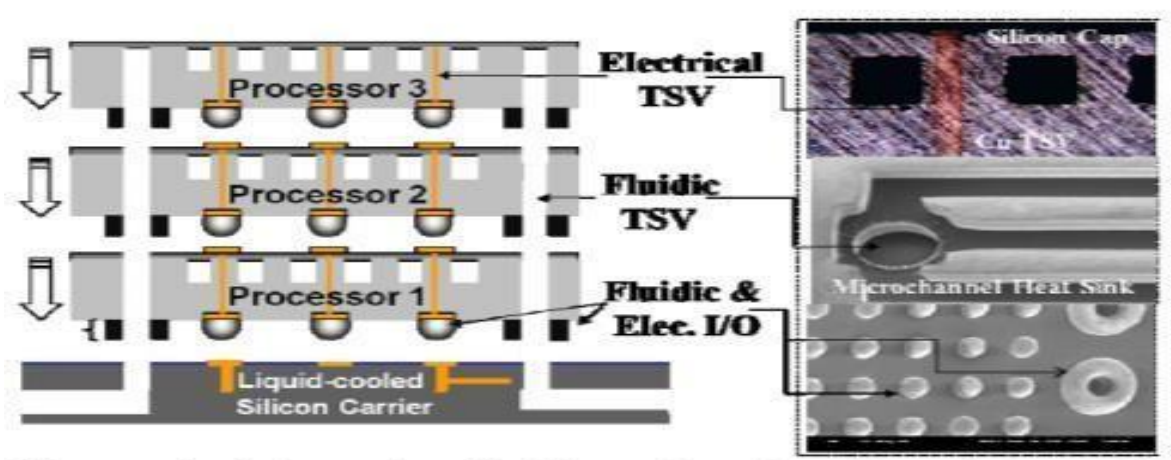

Figure 4. Schematic of chip-scale microchannel heat sink cooling scheme for 3D integrated circuits [5].

Fig. 13 plots the results of temperature measurements at the inlet, outlet, and the average chip temperature when the resistors were heated with a power of $45 \mathrm{~W}$ under flow rates of 65 $\mathrm{ml} / \mathrm{min}$ and $104 \mathrm{ml} / \mathrm{min}$ [4]. The inlet and outlet temperatures were measured by thermocouples directly and recorded in the computer software. It can be seen from Fig. 13 that the on-chip temperature as well as the outlet temperature increase rapidly once power is applied to the 
heaters, while the inlet temperature does not change because the metal block heat exchanger dissipates the power with the aid of the circulating chiller [4]. Under a flow rate of $65 \mathrm{ml} / \mathrm{min}$, the average chip temperature rise is 18.1 , which yields a corresponding thermal resistance of $0.4 \mathrm{C} / \mathrm{W}$. Under a larger flow rate $(104 \mathrm{ml} / \mathrm{min})$, the average temperature rise is 12.7 and the corresponding thermal resistance for the chip is $0.28 \mathrm{C} / \mathrm{W}[4]$.
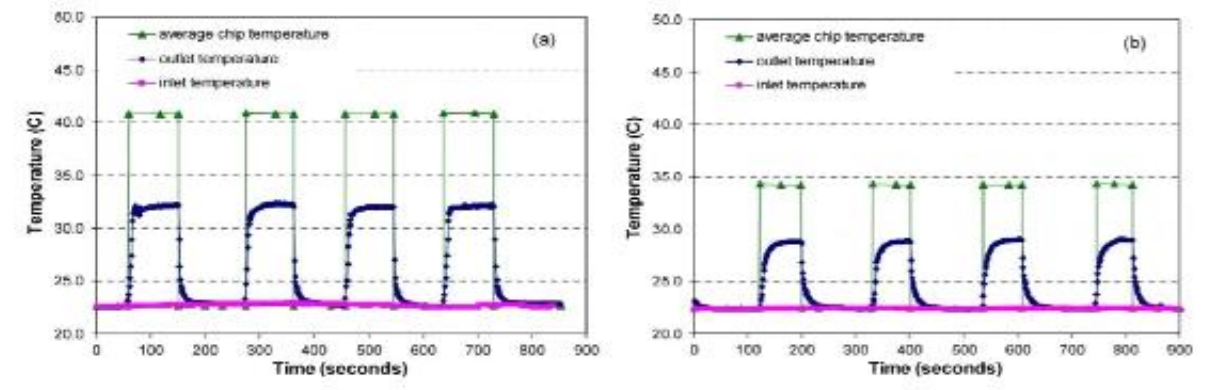

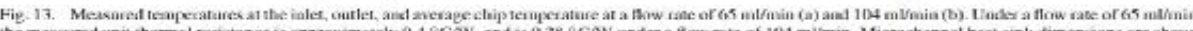

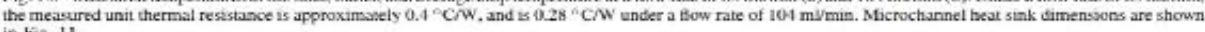
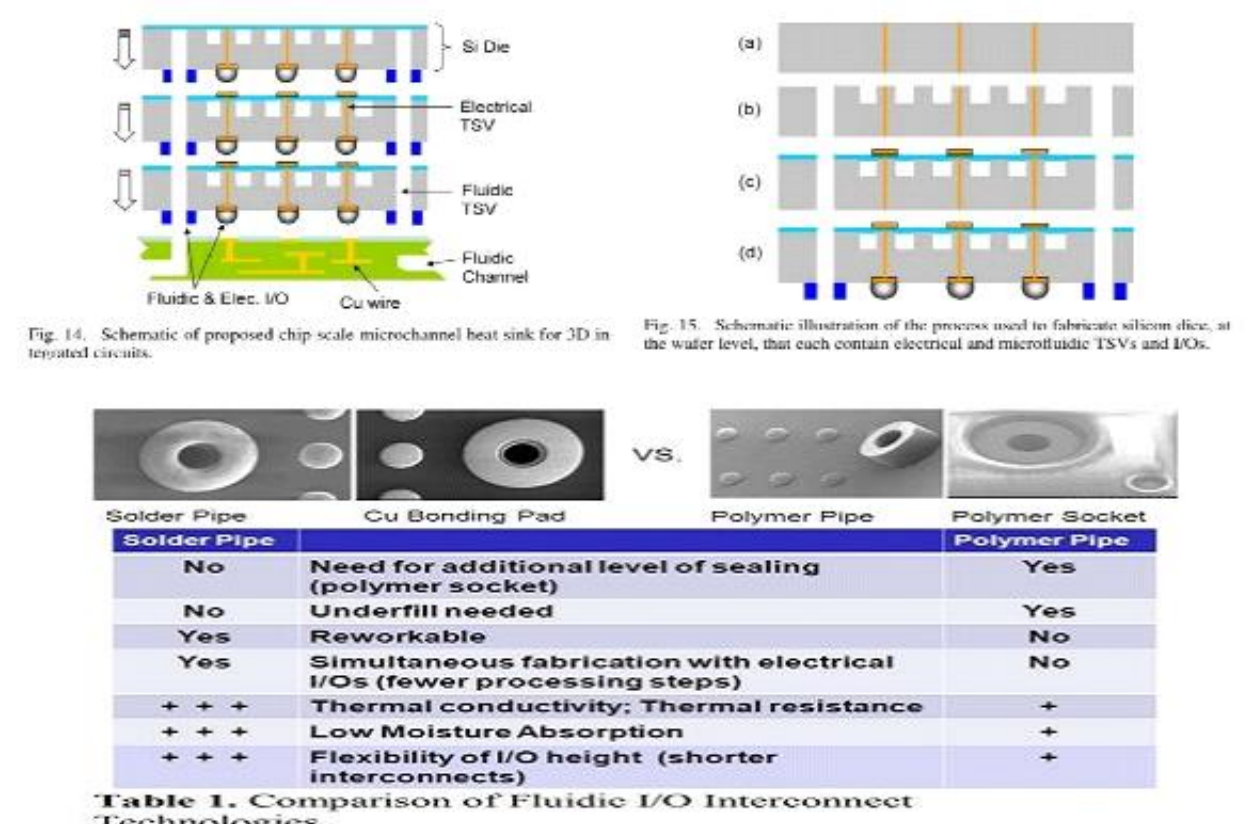

Technologies.

BENEFITS OF MICROCHANNEL COOLED 3D-ICS

\begin{tabular}{|c|c|c|c|}
\hline & Frequency & Power & Temperature \\
\hline $\begin{array}{c}\text { Air cooled } \\
\text { processor } R_{\text {th }}= \\
0.6^{\circ} \mathrm{C} / \mathrm{W}\end{array}$ & $3 \mathrm{GHz}$ & $102 \mathrm{~W}$ & $88^{\circ} \mathrm{C}$ \\
\hline $\begin{array}{c}\text { Microchannel } \\
\text { cooled processor } \\
\mathbf{R}_{\text {th }}=0.24^{\circ} \mathrm{C} / \mathrm{W}\end{array}$ & $3 \mathrm{GHz}$ & $83 \mathrm{~W}$ & $47^{\circ} \mathrm{C}$ \\
\cline { 2 - 4 } & $3.3 \mathrm{GHz}$ & $102 \mathrm{~W}$ & $52^{\circ} \mathrm{C}$ \\
\cline { 2 - 4 } & $4.5 \mathrm{GHz}$ & $254 \mathrm{~W}$ & $88^{\circ} \mathrm{C}$ \\
\hline
\end{tabular}

Here a novel chip scale microfluidic cooling scheme is presented using wafer level batch fabrication and promises to address the cooling and form factor needs. In short, the wafer-level batch fabricated fluidic chip I/Os will be critical to enabling the ultimate performance gains of gigascale silicon integration. 


\section{IMPACT OF LIQUID COOLING}

\subsection{Evaluation Environment}

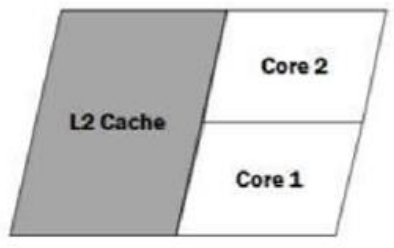

(a)

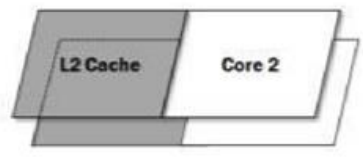

(b)

Fig. 2. (a) Planar layout of cores and an L2 cache, (b) 3D implementation of cores and an L2 cache.

To evaluate power consumption of each functional unit, Wattch and perfmon 2 was used and modified to accommodate the additional performance counter of a Core 2 Duo processor. The Intel Core 2 processor provides three fixed-function performance counters and two generalpurpose performance [1]. Counters are used in the configuration register, which indicates the corresponding performance counter among 129 events 17 events are considered for estimating the activity factor of each functional unit [1]. The coolant goes through in-between the individual dies with the volumetric flow rate (Q) of $6.55 \times 10-3 \mathrm{~m} 3 / \mathrm{hr}$ [1]. The power required for the flowing coolant in-between individual dies is calculated as follows.

$$
P_{\text {flowing_cooling }}=\Delta \text { Pressure } \cdot Q
$$

$\Delta \mathrm{P}$ is the pressure drop calculated from numerical simulation, the forced flowing coolant is only about $0.823 \mathrm{~W}$ due to the low volumetric flow rate, which is enough for the two-die $3 \mathrm{D}$ processor [1]. Compared to the 1 2W power consumption of the conventional air cooling scheme, the forced convective driving power used is reasonable [1].

\subsection{Thermal modelling}

To investigate the heat dissipation capability of the 3D processor with liquid cooling, Fluent Package (ICEPAK) simulation engine is used to analyse heat flow. Each of the die composed of one core and half of the L2 cache is mounted at the board by Flip-chip Ball Grid Array (FBGA) and the components of the package (498-solderball, Flip-chip underfill, and Flip-chip substrate) are modelled as a single combined layer which has equivalent thermal resistance. In the air cooling scheme, the coolant material is the ambient air of $293.15 \mathrm{~K}$ temperature passing through heat spreader and finned heat sink [1]. The constant convective heat transfer coefficient of $15000 \mathrm{~W} / \mathrm{m} 2 \mathrm{~K}$, is sufficiently high enough to represent a well-designed air cooling scheme at the upper side of the heat spreader [1]. In the evaluated liquid cooling scheme, the coolant (293.15K water) flows in a one-dimension in-between the individual dies with a constant mass flow rate. The heat flux of all functional units on each die is considered individually as the heat flux of each functional unit is different depending on its corresponding power consumption. 


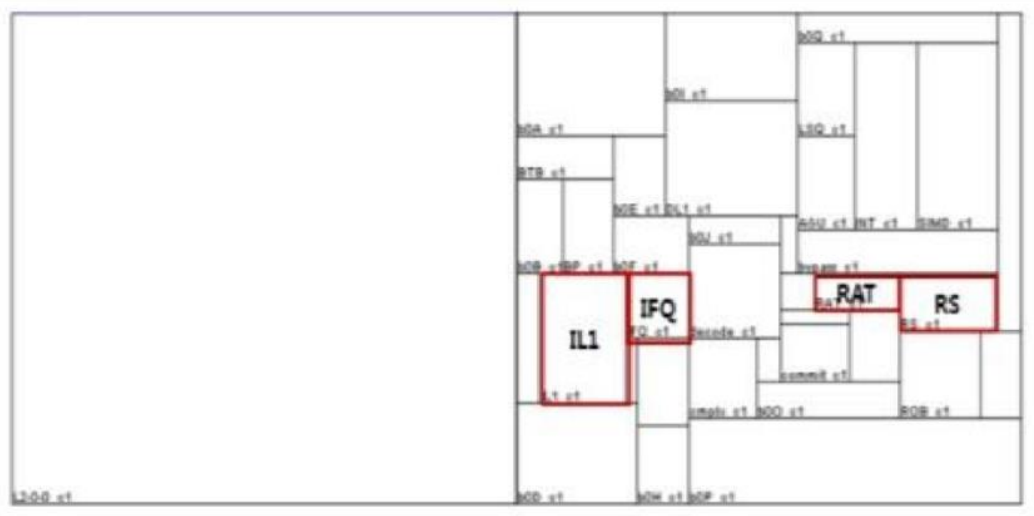

Fig. 3. Floorplan of a die in the 3D integrated processor.

\subsection{Temperature-Dependent Leakage Modelling}

Leakage power mainly consists of subthreshold leakage power and gate leakage power. Two types of leakages are considered, SRAM unit and Logic circuit. The leakage power of the logic circuits is calculated as the product of the number of gates and the average subthreshold leakage current per gate, whereas power of the SRAM units is the sum of SRAM memory cells' leakage power and their peripheral circuits' leakage power. Both the subthreshold leakage power for logic circuits and the gate leakage power for SRAM units are considered for the core 2 Duo processor(65nm) used here [1].

\subsection{Reliability Modelling}

Reliability Aware Microprocessor (RAMP) is used for Lifetime reliability modelling, RAMP can dynamically track the lifetime reliability depending on the application behaviour. This represents the processor lifetime reliability in terms of Mean Time to Failure (MTTF) and calculates an instantaneous MTTF based on the current temperature and the utilization of each functional unit. There are five models in RAMP which are used to quantify the reliability of the 3D chip [1].

$$
\begin{aligned}
& M T T F_{\text {Electro Migration }} \propto\left(J-J_{\text {critical }}\right)^{-N} e^{\frac{\mathrm{E}_{\text {aEM }}}{\mathrm{kT}}} \\
& M T T F_{\text {Stress Migration }} \propto\left|T_{0}-T\right|^{-N} e^{\frac{\mathbb{E}_{a S M}}{\mathrm{kT}}}
\end{aligned}
$$

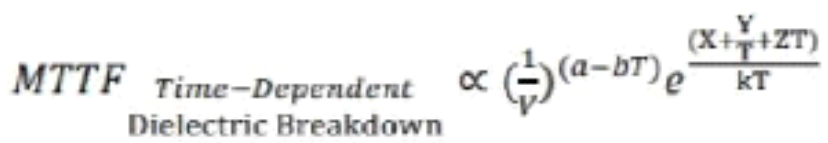

$$
\begin{aligned}
& M T T F_{\text {Thermal cycling }} \propto\left(\frac{1}{T-T_{\text {ambient }}}\right)^{q} \\
& \text { MTTF } \underset{\text { Nemperature Instability }}{\text { Negative Blas }} \propto \\
& {\left[\left(\ln \left(\frac{\mathrm{A}}{1+2 e \frac{\mathrm{B}}{\mathrm{kT}}}\right)-\ln \left(\frac{\mathrm{A}}{1+2 e \frac{\mathrm{B}}{\mathrm{KT}}}-\mathrm{C}\right)\right) \times \frac{\mathrm{T}}{e^{\frac{-1}{\mathrm{kT}}}}\right]^{\frac{1}{\beta}}}
\end{aligned}
$$




\section{RESULT ANALYSIS}

\subsection{Temperature}

The liquid coolant flows from left to right, so the RAT, IFQ \& RS are cooled with a rather warmer liquid.

This can be made much better if the floor plan is changed a bit by placing them on the upper or lower side of L1 cache. As seen the L1 cache in liquid cooling scheme is far cooler than air cooling scheme, when adopting the liquid cooling scheme with the gcc application, average temperature of IL1, IFQ, and RS is reduced by as much as 45 degrees, 35 degrees, and 31 degrees, respectively [1]. In case of the gzip application it is reduced by 42 degrees, 31 degrees and 26 degrees. And volumetric flow rate of $6.55 \times 103 \mathrm{~m} 3 / \mathrm{hr}$, is enough to reduce the temperature of the hottest functional units below the thermal emergency [1].

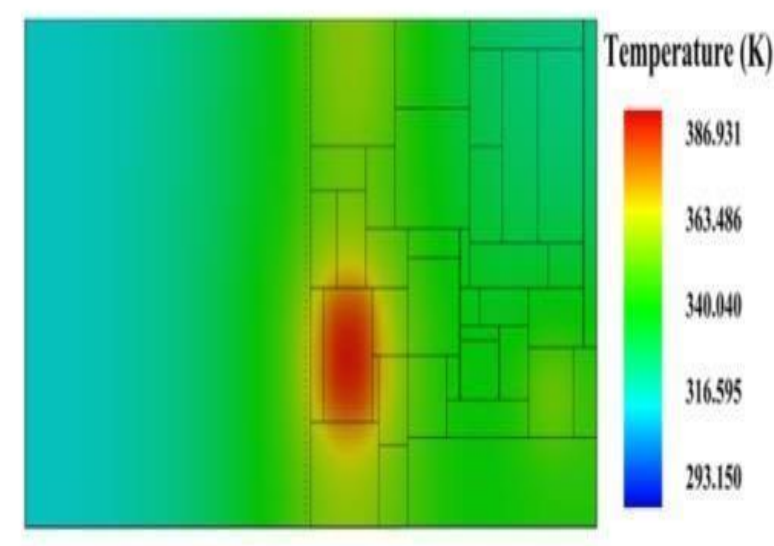

(a) air cooling

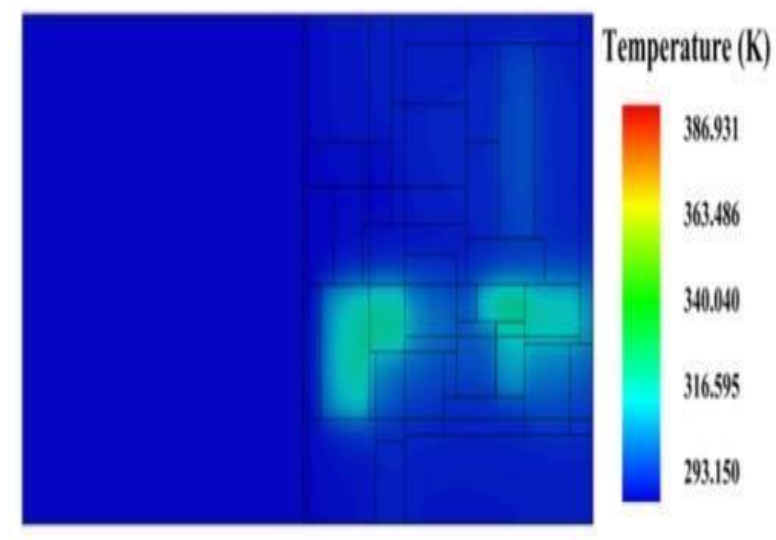

(b) liquid cooling

Fig. 4. Thermal profile of the 3D integrated processor (air cooling vs. liquid cooling)

\subsection{Leakage}

The leakage consumption of three hottest functional units (IL1, IFQ, and RS) is calculated. In gcc application, the liquid cooling scheme reduces the average leakage consumption of IL1, IFQ, and RS by $12.8 \%, 12.7 \%$, and $11.1 \%$, respectively [1]. In case of gzip application, the liquid cooling scheme shows average leakage reduction as $11.5 \%$ (IL1), $11.3 \%$ (IFQ), and 9.4\% (RS), respectively [1]. Since leakage power consumption is dependent on temperature, liquid cooling scheme reduces total processor leakage by $11.5 \%$ and $10.1 \%$, in gcc and gzip [1]. 


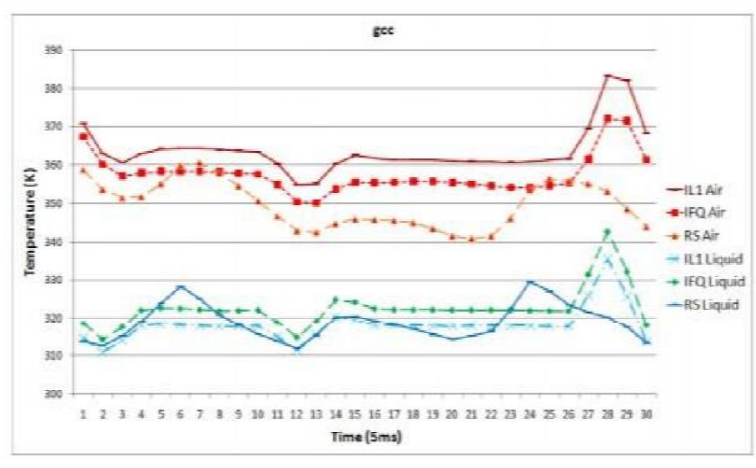

(a) gee

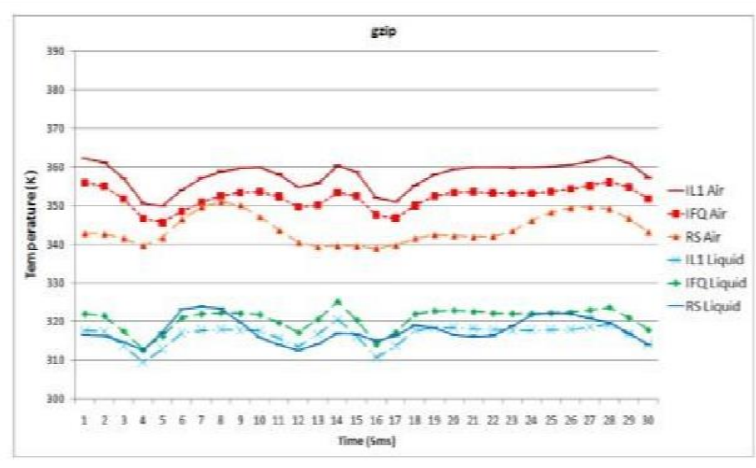

(b) grip

Fig. 5. Temperature comparison of three hottest functional units between the conventional air cooling scheme and the liquid cooling scheme.

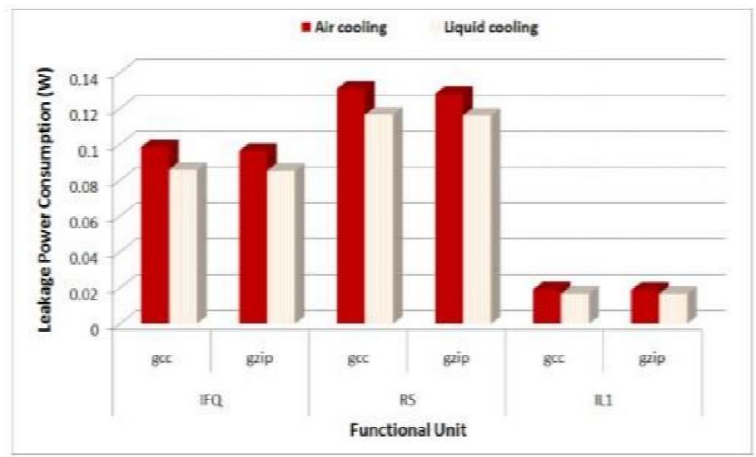

Fig. 6. Leakage consumption of the three hottest functional units.

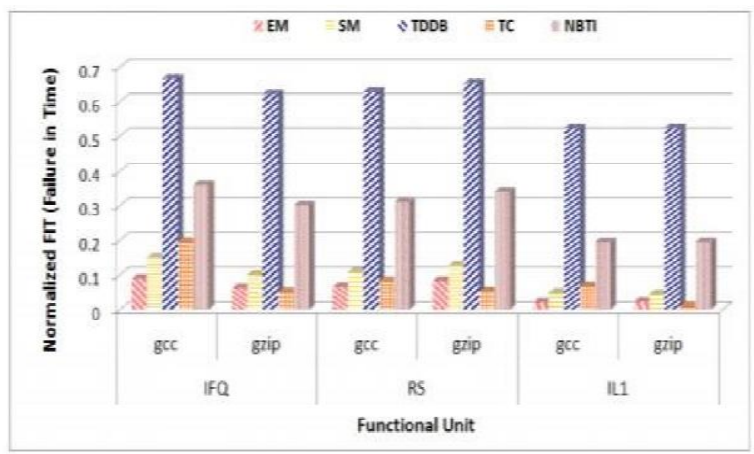

Fig. 7 Normalized FIT values for five different lifetime reliability models.

\subsection{Lifetime Reliability}

3D processor lifetime reliability is calculated in terms of its FIT values. Here, in case of the gcc application, the liquid cooling scheme improves the lifetime reliability of the L1 instruction cache by $97.9 \%, 95.1 \%, 47.6 \%, 93.2 \%$, and $80.5 \%$, corresponding to EM, SM, TDDB, TC, and NBTI, respectively [1]. There are substantial lifetime reliability enhancements with the liquid cooling scheme for the instruction fetch queue and the reservation station since reliability is highly dependent on the difference between the temperature of each functional unit and the ambient temperature of the 3D integrated processor.

\section{CONCLUSION AND FUTURE WORK}

The 3D integration technique provides significant benefits in terms of area, wire length, and power consumption but also increases power density. Here we evaluated the architectural effects (temperature, leakage, and reliability) of the direct interlayer cooling method for the 3D integrated processor, where the dielectric coolant flows inbetween individual dies. liquid cooling scheme significantly reduces on-chip temperature under $350 \mathrm{~K}$, which eliminates thermal emergency [1]. The temperature reduction also leads to more than $10 \%$ leakage reduction and improves the lifetime reliability significantly [1]. So, for the future 3D ICs with both integrated Fluidic \& Electrical TSV are the way to manage the thermals for high density and high-performance chips.

\section{ACKNOWLEDGEMENT}

I would like to express my gratitude toward Dr Ankur Jain for his guidance and constant supervision \& also for his support in completing the project. 


\section{REFERENCES}

[1] Jang, H.B., Yoon, I., Kim, C.H., Shin, S. and Chung, S.W., 2009, October. The impact of liquid cooling on 3D multi-core processors. In Computer Design, 2009. ICCD 2009. IEEE International Conference on (pp. 472-478). IEEE.

[2] Ong, C.L., Paredes, S., Sridhar, A., Michel, B. and Brunschwiler, T., 2014, December. Radial hierarchical microfluidic evaporative cooling for 3-d integrated microprocessors. In Proc. 4th European Conference on Microfluidics, Limerick.

[3] Tang, G.Y., Tan, S.P., Khan, N., Pinjala, D., Lau, J.H., Yu, A.B., Vaidyanathan, K. and Toh, K.C., 2010. Integrated liquid cooling systems for 3-D stacked TSV modules. IEEE Transactions on Components and Packaging Technologies, 33(1), pp.184-195.

[4] Dang, B., Bakir, M.S., Sekar, D.C., King Jr, C.R. and Meindl, J.D., 2010. Integrated microfluidic cooling and interconnects for 2D and 3D chips. IEEE Transactions on Advanced Packaging, 33(1), pp.79-87.

[5] King, C.R., Zaveri, J., Bakir, M.S. and Meindl, J.D., 2010, June. Electrical and fluidic C4 interconnections for inter-layer liquid cooling of 3D ICs. In Electronic Components and Technology Conference (ECTC), 2010 Proceedings 60th (pp. 1674-1681). IEEE. 